
cultures

Les cahiers de l'Acedle

\title{
Réalité et perspectives de l'enseignement / apprentissage du français de spécialité à l'université en Albanie
}

\section{Esmeralda Kromidha}

\section{OpenEdition}

1 Journals

Édition électronique

URL : http://journals.openedition.org/rdlc/2886

DOI : $10.4000 /$ rdlc.2886

ISSN : 1958-5772

Éditeur

ACEDLE

\section{Référence électronique}

Esmeralda Kromidha, «Réalité et perspectives de l'enseignement / apprentissage du français de spécialité à l'université en Albanie », Recherches en didactique des langues et des cultures [En ligne], 3 | 2008, mis en ligne le 30 juillet 2008, consulté le 20 avril 2019. URL : http://journals.openedition.org/ rdlc/2886 ; DOl : 10.4000/rdlc.2886

\section{Ce document a été généré automatiquement le 20 avril 2019}

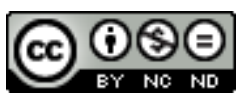

Recherches en didactique des langues et des cultures is licensed under a Creative Commons AttributionNonCommercial-NoDerivatives 4.0 International License 


\title{
Réalité et perspectives de l'enseignement / apprentissage du français de spécialité à l'université en Albanie
}

\author{
Esmeralda Kromidha
}

\section{Le cadre institutionnel}

1 En Albanie la formation des enseignants est exclusivement linguistique et littéraire. On insiste sur l'apprentissage des compétences linguistique, communicative et culturelle en français dit général (vie quotidienne, pratiques sociales et culturelles). Un séminaire de didactique est proposé en troisième et quatrième année. L'accent y est mis sur une analyse théorique et pratique de l'enseignement / apprentissage de la langue étrangère générale à un public du primaire et du secondaire.

2 Mais comme D. Lehman, (1993 : 58) l'indique: «Sous la pression des évolutions à la fois technologiques et politico - économiques, un besoin latent de formation linguistique plus ou moins spécialisée s'est créé selon le cas ».

3 Les bouleversements qui ont affecté l'Europe ces dernières années ne manquent pas de produire des effets sur les politiques éducatives en Albanie. On perçoit la nécessité d'un enseignement fonctionnel du français et on fait des efforts en ce sens depuis 1994. La formation des enseignants reste le facteur principal pour mener à bien cette tâche. L'ambassade de France en Albanie joue un rôle important par des politiques de coopération et de formation d'enseignants, des bourses d'études en France, des séminaires de formation. 


\section{Les caractéristiques du public étudiant albanais}

4 Les étudiants des facultés de la capitale, Tirana, viennent de différentes régions du pays. Ils ont 18 ou 19 ans. Après leurs études secondaires, ils passent des examens avant de commencer leurs études supérieures. Ils sont obligés de choisir la langue étrangère qu'ils ont étudiée au lycée. Leur niveau est censé être B2 (Cadre Européen Commun de Référence pour les Langues). Le cours de langue étrangère à la faculté se déroule pendant les deux premières années à raison de 120 séances de 50 minutes. Dans les cours de français les domaines de spécialité des étudiants sont homogènes.

D. Lehman $(1993: 58)$ affirme que

la particularité de ces publics est qu'ils ne présentent pas de besoins tels qu'ils puissent à proprement parler constituer des objectifs spécifiques réels. Publics universitaires engagés dans des études qui ne sont pas le français, ils ont néanmoins à leur programme une langue étrangère, et ils se trouvent avoir opté pour le français (sans que ceci résulte toujours d'un choix libre et délibéré). Mais rien dans leur environnement langagier immédiat ne donne à penser qu'ils aient à communiquer en français en dehors des cours eux-mêmes (...). L'institution a décidé pour eux que, puisqu'ils sont étudiants en droit, en médecine ou en anthropologie, ils feraient $\mathrm{du}$ français $\mathrm{du}$ droit, de la médecine ou de l'anthropologie.

On peut partager ce point de vue pour ce qui concerne l'Albanie avant son ouverture vers le monde occidental. La langue était apprise avec pour seul but d'être «cultivé ». Aujourd'hui les changements socio-économiques récents influencent parfois les motivations des étudiants; les conceptions de la langue et de son apprentissage peuvent être plus variées.

7 Les étudiants albanais deviennent peu à peu conscients de l'utilité de la langue étrangère. Ainsi par exemple, pour un étudiant qui va devenir un professionnel du tourisme, il est tout a fait imaginable de se trouver en contact avec des francophones dans l'exercice de son métier.

Dans une telle situation, nous sommes d'avis que

mieux les connaître, c'est mieux les servir, mais pour mieux les servir il faut être prêt à diversifier les approches. La langue que nous enseignons n'est plus alors conçue comme un tout, objet immuable, dont il faut connaître tous les arcanes pour oser l'utiliser, les parcours sont diversifiés, les compétences visées parcellisées, partielles, peut-être plus vite acquises. Plus vite parce que la notion de temps prend toute son importance pour ces publics, peu disponibles et pressés de voir leur effort couronné de succès (Cheval, $2003: 12)$.

Il convient de

«...s'interroger sur ce que l'on attend de l'apprenant à l'issue de sa formation, sur les utilisations concrètes qu'il devra faire de la langue française. L'apprentissage de la langue est donc, fondamentalement, défini par son utilité présente et future » (Carras et al., 2007: 22).

10 Nous avons tenté de mieux connaître les besoins et les objectifs des étudiants des différentes filières et des enseignants qui enseignent le français langue étrangère à l'université. 


\section{L'enseignement du français de spécialité - deux questionnaires}

11 Nous avons proposé des questionnaires aux enseignants de FLE de différentes facultés de Tirana et aux étudiants en voie de spécialisation de ces facultés. Nous avons recueilli les réponses de dix enseignants et de 220 étudiants de première et deuxième année. Notre but était de faire émerger les images et les attentes des sujets interrogés vis-à-vis du français sur objectifs spécifiques et de dégager ensuite des amorces de solutions.

\section{Les réponses des enseignants}

12 Les enseignants sont jeunes dans leur profession, avec une expérience de 2 ou 3 années, à l'exception d'une enseignante qui a une expérience professionnelle de 11 années. Ils enseignent dans les facultés des sciences sociales et politiques, d'économie et management du tourisme, de médecine et de géographie et utilisent tous un manuel de français général : Le nouveau sans frontières (vol. 1, 2) ou Bonne Route (vol. 1, 2). Aucun ne possède de manuel de français de spécialité. Les enseignants essaient de trouver des documents authentiques correspondant aux spécialités mais il ne leur est pas facile de les adapter au niveau souvent peu avancé des étudiants. La difficulté liée à la connaissance de la terminologie des textes spécialisés est mentionnée. Les enseignants les plus jeunes n'ont pas de connaissances dans les spécialités de leurs étudiants car leur formation a été seulement linguistique et littéraire. L'enseignante plus expérimentée indique qu'elle a acquis des connaissances au fur et à mesure de l'exercice de son métier. Les enseignants préféreraient tous le travail avec un manuel de français de spécialité et des documents authentiques. L'enseignement de la langue de spécialité est limité à des termes de la spécialité. La seule activité mentionnée consiste à faire apprendre les termes du domaine en donnant des listes de termes ou des textes et en demandant de trouver une traduction dans le dictionnaire.

\section{Les réponses des étudiants}

13 Tous les étudiants affirment qu'ils ont été obligés de choisir le français dès leurs études secondaires. Une majorité (185 étudiants) répond qu'elle étudie le français pour le pratiquer dans son futur métier. Certains (10 étudiants) envisagent de faire des études en France, d'autres (25 étudiants) parlent de lire des bibliographies qui existent en Français. Presque tous considèrent que l'apprentissage du français signifie un enrichissement culturel. La totalité des étudiants mentionne l'utilisation de manuels de français général, un petit nombre ajoute l'utilisation de documents authentiques liés au domaine de spécialité.

14 Presque tous les étudiants de deuxième année affirment qu'ils ne peuvent pas communiquer en langue de spécialité puisque la base a été un manuel de français général et que les termes techniques constituent une vraie difficulté. Certains mentionnent que, même au niveau du français général, ils ne peuvent pas communiquer facilement pendant les cours de français, et que quelquefois ils font de nouveau ce qui a été fait au lycée, une répétition ennuyeuse pour ceux qui espéraient de nouvelles connaissances à ce stade d'études. La plus grande partie éprouvent des difficultés en compréhension et expression 
orales (170 étudiants); d'autres se heurtent à des difficultés en expression écrite (50 étudiants). Pour plus de la moitié des étudiants, l'expression orale constitue la compétence la plus importante, mais ils ne sous-estiment pas l'écrit. Ils préfèrent l'oral parce qu'ils veulent communiquer facilement après leurs études. Les étudiants répondent qu'ils n'ont pas une formation suffisante dans leur domaine de spécialité. Seuls deux d'entre eux indiquent qu'ils ont été en France pendant deux ans. Ils ne sont pas satisfaits des horaires : ils ne disposent pas d'assez de temps pour l'apprentissage de la langue de spécialité avant de la pratiquer. La communication en français pendant le cours leurs a beaucoup manqué. Ils souhaiteraient l'utilisation de supports audio-visuels pour que le programme soit mieux adapté à leurs besoins. Ils demandent que les cours de français soient plus adaptés à leur domaine de spécialité. La majorité demande un changement de manuel et une plus grande activité de communication en français de spécialité pendant le cours ainsi que l'emploi de documents authentiques de la réalité française.

\section{Trois grands problèmes à relever}

Comment former les enseignants albanais de français à l'enseignement dans les facultés spécialisées? Lorsqu'il est demandé à ces enseignants, avec une formation linguistique et littéraire d'enseigner le français de la médecine, de la chimie, du droit ou d'autres domaines de spécialité, se pose la question de la connaissance du domaine visé. Dans ces conditions l'enseignant doit être un excellent connaisseur du français général et un enseignant expert des termes et des situations de communication en usage dans telle ou telle spécialité en même temps qu'un enseignant très bien formé en pédagogie et didactique des langues. Nous ne pourrons pas traiter ce problème en détail ici mais nous donnerons quelques propositions au niveau de la formation des enseignants.

Le manque de manuels de français de spécialité adaptés au contexte albanais. Un enseignement sur objectifs spécifiques ne peut se faire de manière satisfaisante dans cette situation.

Compte tenu du fait que les étudiants ne sont pas satisfaits de l'enseignement qu'ils subissent, apparaît un problème méthodologique: Comment bâtir un parcours d'enseignement de FS efficace tenant compte de la réalité albanaise?

\section{La motivation - paramètre essentiel dans la situation actuelle d'enseignement / apprentissage en Albanie}

Dans le questionnaire, nous avons relevé qu'un certain nombre d'étudiants n'éprouvent pas une forte motivation pour apprendre le français. Beaucoup d'apprenants suivent des cours de langue dans le seul but d'avoir une note suffisante pour être reçus à l'examen final.

Si on décide de placer l'apprenant au cœur de l'apprentissage, il faut d'abord comprendre sa motivation, si elle existe.

l'apprenant devrait avoir la latitude de sélectionner des informations supplémentaires, de choisir son propre rythme d'apprentissage en fonction des besoins, en d'autres mots, il devrait pouvoir façonner son propre syllabus et 
développer des stratégies d'apprentissage personnelles. Cette implication de

l'apprenant devrait en principe résoudre le problème de la motivation.

21 Nous partageons cet avis mais il n'est pas toujours aisé pour un enseignant, si déterminé soit-il, de modifier la relation pédagogique et de garantir la motivation de l'apprenant. Modifier l'attitude de l'apprenant, les représentations qu'il s'est forgées est une tâche complexe. La motivation d'un public captif est tributaire non seulement du cadre institutionnel, mais aussi de l'image qu'il a de la langue et du contexte économique et politique (la demande sur le marché du travail, politiques linguistiques, etc.).

\section{L'enseignant de FLE face au lexique spécialisé}

Il faut trouver une réponse au problème « oiseux et sempiternel de savoir si l'enseignement doit être assuré par un professeur de français initié à la discipline visée ou par un professeur de cette discipline initié à l'enseignement du français » (Porcher, 1976 : 74).

La question du lexique spécialisé semble un point important dans la mesure où les enseignants et futurs enseignants de FLE se la posent immédiatement. Leurs inquiétudes ne sont que partiellement levées par les réponses que l'on trouve dans les ouvrages s'interrogeant sur les compétences nécessaires à l'enseignant de français. Il y est généralement précisé qu'il ne s'agit pas pour l'enseignant de devenir spécialiste du domaine. Ainsi, P. Lerat, (1995 : 21) invite à considérer la notion de langue spécialisée non comme un recueil de termes plus ou moins abrupts mais comme « une langue en situation d'emploi professionnel ». L'enseignant de FS reste alors avant tout un professeur de français, réceptif à un certain nombre de situations et de logiques de travail. Il suffit de bien distinguer entre les compétences linguistiques et la maîtrise du domaine et on peut affirmer, à l'instar de 0 . Challe, (2003:19) que

la maîtrise du savoir se dédouble en deux connaissances: les connaissances linguistiques et les connaissances du domaine de spécialité. Certes le formateur possède mieux la langue française que l'étudiant. À l'inverse, l'étudiant connaît mieux le domaine que son professeur. Il s'agit en ce cas de trouver un nouvel équilibre.

L'adoption d'une vision de la langue de spécialité ne dispense vraisemblablement pas d'un début de spécialisation dans le domaine, mais cela suppose une sensibilisation aux différentes logiques, linguistique, disciplinaire et professionnelle.

Comme nous l'avons précisé, en Albanie, les textes littéraires constituent le noyau dur de la formation. Les futurs enseignants sont rarement confrontés aux réalités politiques, économiques, à la législation, à la structure de l'entreprise, etc. et ont une connaissance disciplinaire incomplète par rapport à la demande des différentes filières universitaires.

Naturellement, il ne s'agit pas de former des experts en économie, commerce, médecine, etc., mais plutôt de familiariser les futurs professeurs à des documents autres que les textes des écrivains, des cultures plus en prise avec la réalité quotidienne ou le monde des professions. Il s'agit donc d'une sensibilisation à une conception élargie de la notion de culture, et à d'autres domaines que la littérature ou l'histoire. On pourrait espérer un effet bénéfique en contribuant à nuancer les représentations de la langue culture étrangère et en permettant d'atténuer le sentiment d'insécurité ou de malaise face à l'enseignement de la langue de spécialité.

D'après D. Lehmann, (1993:13) : 
l'enseignant de français de spécialité devra d'abord mener à bien une acculturation personnelle (intraculturelle si l'on veut) avant d'être en mesure de favoriser chez les apprenants avec qui il travaillera une autre acculturation, inter-culturelle cellelà. [...] On est souvent tenté de penser que le peu de goût manifesté par les enseignants pour l'enseignement du français aux publics spécifiques tient pour beaucoup à leur crainte face aux mystères que l'univers scientifique et technique étale à leurs yeux de littéraires.

Pour cette raison il conseille de multiplier les outils de formation des enseignants destinés à la favoriser. Sans pour autant devenir spécialiste, l'enseignant de langues doit disposer d'un minimum de connaissances scientifiques.

\section{Conclusion}

Les étudiants ont des besoins langagiers professionnels ou d'études auxquels les formations doivent répondre avec efficacité. En ce qui concerne la formation des enseignants, il nous paraît nécessaire de mettre en place des formations continues. Ces formations pourraient avoir comme objectif dans un premier temps le recueil et l'exploitation des corpus de discours du français de spécialité afin de mieux comprendre leur fonctionnement puis, dans un deuxième temps, de réfléchir sur l'élaboration de programmes de FS et d'outils pédagogiques.

\section{BIBLIOGRAPHIE}

Carras, C., Tolas, J., Kohler, P.\& Szilagyi, E. (2007). Le français sur objectifs spécifiques et la classe de langue. Paris. CLE international.

Challe, O. (2002). Enseigner le français de spécialité. Paris : Economica.

Conseil de l'Europe (2001). Cadre Européen Commun de Référence pour les Langues. Paris : Didier.

Lehmann, D. (1993). Objectifs spécifiques en langue étrangère. Paris : Hachette.

Lerat, P. (1995). Les langues spécialisées. Paris : PUF.

Porcher, L. (1976). « Monsieur Thibaut et le Bec Bunsen ». Études de linguistique appliquée, no 23, juillet- septembre. p.77.

Porcher, L. (2002). «Y a-t-il un français sans objectif(s) spécifique(s) ? ». Les cahiers de l'Asdifle, n $\circ 14$.

\section{RÉSUMÉS}

Cet article est une réflexion critique sur le milieu universitaire en Albanie. Nous partirons d'une description pour terminer avec la formation de l'enseignant de FS. 
This paper is a critical reflection on the Albanian University. We will start with a description and end with the concept of the professional culture of the French teacher.

\section{INDEX}

Keywords : teaching, French for specific purposes, university, Albania

Mots-clés : enseignement, français de spécialité, université, Albanie

\section{AUTEUR}

\section{ESMERALDA KROMIDHA}

Université de Tirana, Albanie

Esmeralda KROMIDHA est maître-assistante au département de français de la faculté des langues étrangères de Tirana.

Courriel : aldanushi[at]yahoo.fr 